๑๑ Entomologica Fennica. 9 December 2002

\title{
Index to genera and species, Volume 13 (2002)
}

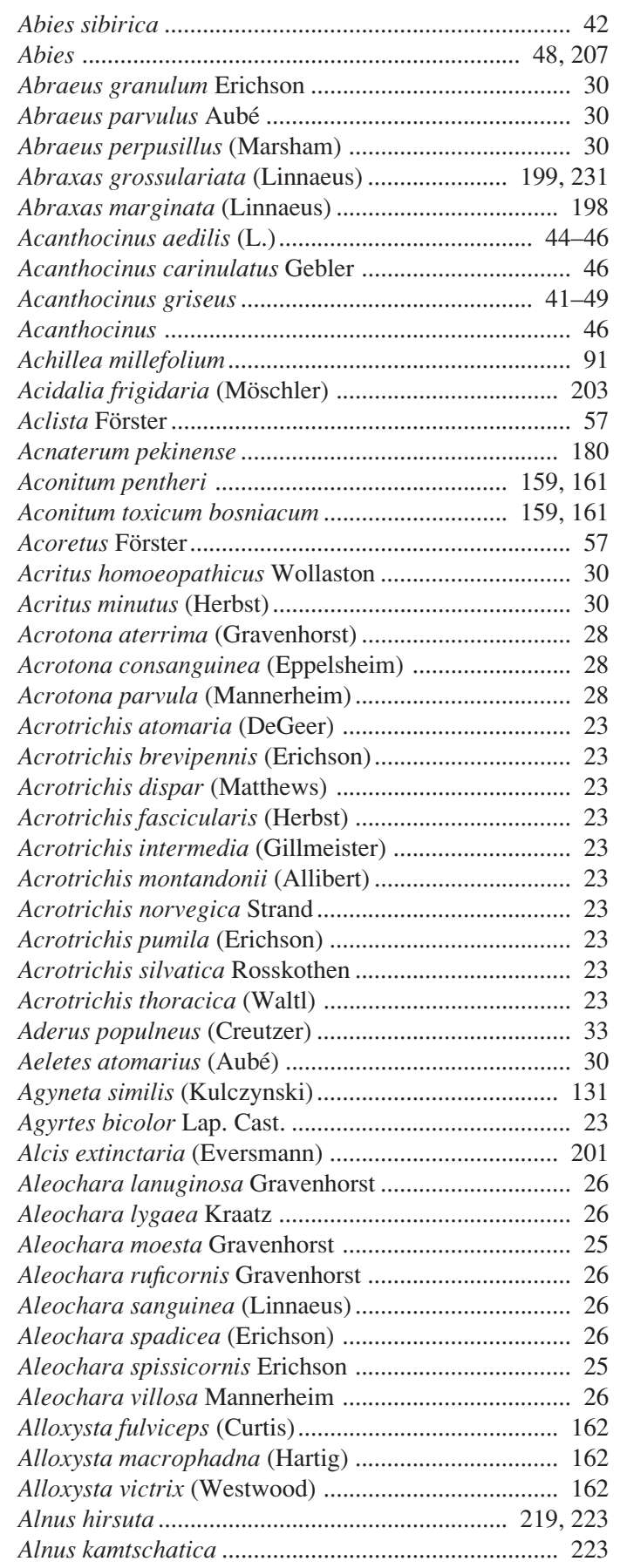

Alnus ................................ 207, 211, 213, 221, 222, 227

Aloconota sulcifrons (Stephens) ................................. 27

Alopecosa aculeata (Clerck) ................................... 131

Amarochara bonnairei (Fauvel) ................................ 27

Amarochara forticornis (Lacordaire) .......................... 27

Amarochara umbrosa (Erichson) .............................. 27

Amauronyx maerkeli (Aubé) ....................................... 30

Amischa analis (Gravenhorst) .................................. 28

Amischa bifoveolata (Mannerheim) ............................ 28

Amischa nigrofusca (Stephens) .................................. 28

Ampedus balteatus (Linnaeus) ................................... 31

Ampedus cinnabarinus (Eschscholtz) ......................... 31

Ampedus hjorti (Rye) ............................................ 31

Ampedus pomorum (Herbst) ....................................... 31

Ampedus rufipennis (Stephens) ................................. 31

Amphigerontia flavonimbatas [sic!] (Rostock) ........... 59

Amphigerontia ....................................................... 58

Amphotis marginata (Fabricius) ................................. 31

Anarta Ochsenheimer .................................................. 1

Andromeda polifolia .................................................... 131

Anectata Förster...................................................... 57

Anevrina curvinervis .............................................. 127

Anevrina ................................................................ 126

Anisarthrocera Semenov .......................................... 105

Anotylus rugosus (Fabricius) ..................................... 25

Aphidius ervi Hal. ................................................. 162

Aphidius sussi Pennacchio \& Tremblay .................... 162

Archiearis parthenias sajana (Prout, 1912) .............. 197

Arctocorisa chanceae Hungerford .............................. 86

Arctocorisa convexa (Fieber) .................................. 86

Arctocorisa planifrons (Kirby) .................................... 86

Arctostaphylos uva-ursi .................................... 2, 5, 6

Artocorisa sutilis (Uhler) ........................................... 87

Asaphodes taigana (Djakonov) ................................. 217

Ascotis selenaria (Denis \& Schiffermüller) ............... 231

Aspitates orciferarius (Walker) ............................... 128

Aspitates taylori sibirica (Djakonov) ......................... 202

Astenus gracilis (Paykull) .......................................... 25

Asthenargus helveticus Schenkel .............................. 131

Asthenargus paganus (Simon) ................................. 131

Atheta brunnea (Fabricius) .......................................... 28

Atheta brunneipennis (Thomson) ................................ 28

Atheta castanoptera (Mannerheim) .............................. 28

Atheta confusa (Märkel) .......................................... 28

Atheta flavipes (Gravenhorst) .................................... 27

Atheta fungi (Gravenhorst) ........................................ 27

Atheta fungicola (Thomson) ....................................... 28

Atheta hepatica (Erichson) ....................................... 28

Atheta longicornis (Gravenhorst) ............................... 28

Atheta myrmecobia (Kraatz) ....................................... 27

Atheta nigra (Kraatz) ........................................... 27 
Atheta scapularis (Sahlberg) ....................................... 27

Atheta sodalis (Erichson) ............................................... 27

Atheta subterranea (Mulsant \& Rey) ........................... 28

Atheta talpa (Heer) …………………………………...... 27

Atheta trinotata (Kraatz) .............................................. 27

Atholus corvinus (Germar) ............................................. 30

Atomaria nigriventris Stephens ................................... 32

Atomaria peltata Kraatz ............................................... 32

Atomaria procerula Erichson ......................................... 32

Atriplex ............................................................... 113

Batrisodes adnexus (Hampe) ....................................... 30

Batrisodes delaporti (Aubé) ............................................ 30

Batrisodes hubenthali Reitter .................................... 30

Batrisodes venustus (Reichhenbach) ............................ 30

Bellardia pubicornis Zett. .......................................... 101

Bellardia stricta (Vill.) ................................................ 101

Betula ........... 56, 199-202, 207, 217, 220-222, 225, 227

Betula ermanii ................................... 212, 216, 219, 223

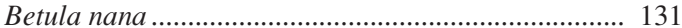

Betula pendula …………………………………...... 82

Betula platyphylla ……………………………….... 220

Betula pubescens ……………………………………... 94

Billaea triangulifera (Zetterstedt) ................................. 46

Bisnius subuliformis (Gravenhorst) .............................. 24

Bledius procerulus Erichson .......................................... 25

Boarmia extinctaria (Eversmann) ............................. 201

Bolitobius cingulatus Mannerheim ................................. 25

Bolyphantes luteolus (Blackwall) ............................... 131

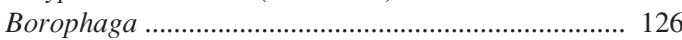

Botrychium boreale ……………………………..... 91

Botrychium lunaria ...................................................... 91

Brachygluta fossulata (Reichhenbach) .......................... 30

Brephos parthenias (Linnaeus, 1761) ......................... 197

Bryaxis curtisii (Leach) ............................................... 30

Cabera exanthemata exanthemata (Scopoli) .............. 202

Caenoscelis ferruginea (Sahlberg) ............................... 32

Caenoscelis sibirica Reitter .......................................... 32

Calamagrostis arundinacea ....................................... 180

Callicerus obscurus Gravenhorst ................................ 27

Callicerus rigidicornis Erichson ................................... 27

Callicorixa alaskensis Hungerford ............................... 86

Callicorixa audeni Hungerford ..................................... 86

Calliphora genarum Zett. ........................................... 101

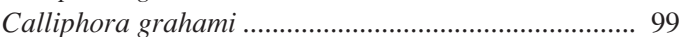

Calliphora loewi End. .......................................... 99-101

Calliphora stelviana B. \& B..................................... 101

Calliphora subalpina Ringd. ................................ 99, 100

Calliphora uralensis Vill................................... 99, 100

Calliphora vicina R.-D. ........................................ 99, 100

Calliphora vomitoria $\mathrm{L}$............................... 99, 100, 102

Calluna vulgaris ....................................................... 2, 6

Calothysanis rectistrigaria (Eversmann) .................... 202

Camponotus …………………………………….... 35

Camponotus herculeanus (Linnaeus) ............................ 34

Camponotus ligniperda (Latreille) ................................ 34

Camponotus vagus (Scopoli) ........................................ 34

Cantharis livida Linnaeus ....................................... 31

Capricornia boisduvaliana (Duponchel) ............... 90-96
Cardiophorus asellus Erichson .................................... 31

Carex ......................................................... 131

Carphacis striatus (Olivier) ........................................ 25

Carsia paludata (Thunberg) ........................................ 230

Carsia sororiata (Hübner) ......................................... 230

Cenocorixa utahensis (Hungerford) .............................. 86

Cerocoma (Cerocomina) Kaszab ......................... 106, 111

Cerocoma (Meloides) Piller \& Mitterpacher............... 106

Cerocoma (Mesocerocoma) Kaszab ............................. 106

Cerocoma (Metacerocoma) Kaszab .................... 106, 111

Cerocoma Geoffroy ..................................... 105, 106, 111

Cerocoma prevezaensis Dvorak ......... 106, 107, 110, 111

Cerocoma schaefferi (L.) .................... 106, 107, 110, 111

Cerocoma schreberi Fabricius ........................... 105-111

Cerocoma vahli Fabricius ........................... 106, 107, 111

Cerylon ferrugineum Stephens ...................................... 32

Cerylon histeroides (Fabricius) .................................... 32

Cetonia aurata (Linnaeus) .............................................. 31

Chennium bituberculatum Latreille .............................. 30

Chenopodium ............................................................. 113

Chiasmia clathrata clathrata (Linnaeus) .................... 198

Chiasmia clathrata djakonovi Kardakoff ................... 199

Cidaria (Calostigria) aptata (Hübner) ........................ 217

Cidaria (Dasyuris) polata (Duponchel) ...................... 210

Cidaria (Entephria) caesiata norvegica (Strand) ....... 210

Cidaria (Epirrhoë) alternata (Müller) ........................ 210

Cidaria (Epirrhoë) hastulata reducta Djakonov ........ 209

Cidaria (Eulype) subhastata clarior Djakonov .......... 221

Cidaria (Euphyia) silaceata deflavata Staudinger .... 212

Cidaria (Euphyia) silaceata leuca Djakonov ............. 212

Cidaria (Lampropteryx) suffumata arctica Sparre-Schneider

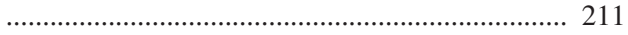

Cidaria (Lampropteryx) suffumata defumata (Stichel) .....

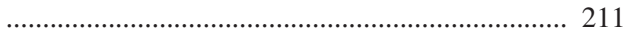

Cidaria (Psychophora) frigidaria (Guenée) .............. 223

Cidaria (Xanthorhoe) biriviata (Borkhausen) ............ 203

Cidaria (Xanthorhoe) designata (Hufnagel) ............... 203

Cidaria (Xanthorhoe) munitata (Hübner) .......... 204, 208

Cidaria (Xanthorhoe) paramushira Bryk .................. 208

Cidaria (Xanthorhoe) spadicearia (Denis \& Schiffermüller)

Cidaria aternata (Minler)... 204

Cidaria alternata (Müller) ......................................... 210

Cidaria cognata (Thunberg).............................. 217, 232

Cidaria ferrugata (Clerck) ......................................... 207

Cidaria ferrugata malaisei Djakonov ......................... 207

Cidaria ferrugata spadicearia (Borkhausen) .............. 204

Cidaria fluctuata (Linnaeus) ...................................... 207

Cidaria fulvata (Forster) .................................... 216, 232

Cidaria furcata (Thunberg) ......................................... 218

Cidaria hastata (Linnaeus) .......................................... 220

Cidaria infuscata (Tengström) ...................................... 216

Cidaria kamtshatica Djakonov .......................... 208, 209

Cidaria luctuata albidior (Alpheraky) ........................ 220

Cidaria luteata Choi .......................................... 216, 232

Cidaria otregiata (Metcalfe) ..................................... 211

Cidaria ruberata (Freyer) .......................................... 218

Cidaria sociata (Borkhausen) ..................................... 210

Cidaria taeniata (Stephens) ........................................ 224 


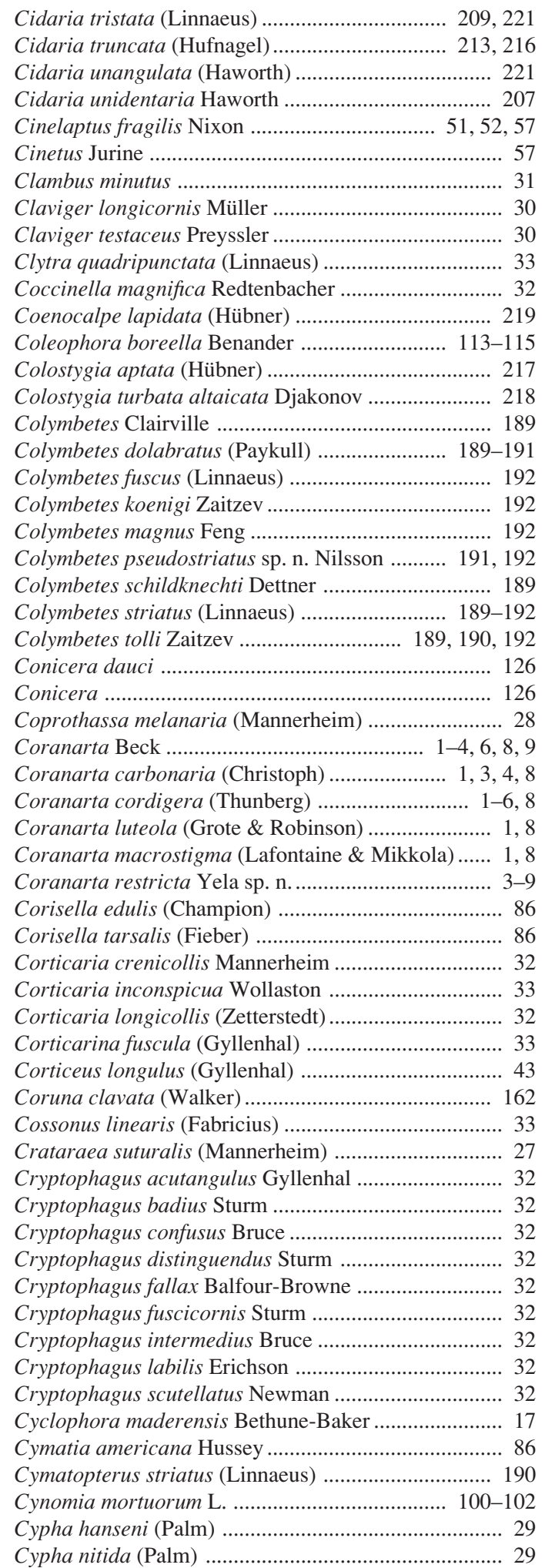

Cypha pulicaria (Erichson) ......................................... 29

Dasycorixa hybrida (Hungerford) ............................... 86

Dasycorixa johanseni (Walley) ..................................... 86

Dasycorixa …...................... 87

Delphinobium junackianum Karsch .................. 161, 162

Dendrophilus corticalis (Paykull) ............................... 30

Dendrophilus pygmaeus (Linnaeus) ............................... 30

Dermestes palmi Sjöberg ............................................ 31

Diastictus vulneratus (Sturm) ........................................ 31

Dienerella clathrata (Mannerheim) ............................... 32

Dienerella elongata (Curtis) ......................................... 32

Dienerella ruficollis (Marsham) ..................................... 32

Dinarda dentata (Gravenhorst) .................................... 27

Dinarda hagensii Wasmann ....................................... 27

Dinarda maerkelii Kiesenwetter .................................. 27

Diplocentria bidentata (Emerton) ............................. 131

Dolichomitus terebrans (Ratzeburg) .............................. 46

Dorcatoma ambjoerni Baranowski ...................... 82, 83

Dorcatoma androgyna Büche ...................................... 83

Dorcatoma chrysomelina Sturm .............. 81-83, 116, 117

Dorcatoma dresdensis Herbst .................... 116, 118-120

Dorcatoma flavicornis (Fabricius) ...................... 82, 117

Dorcatoma Herbst ................... 79, 82, 84, 116, 117, 120

Dorcatoma janssoni Büche \& Lundberg sp. n. ...... 79-83

Dorcatoma lanuginosa Baudi ......................................... 84

Dorcatoma lomnickii Reitter ............................. 120, 121

Dorcatoma minor Zahradnik ......................... 82, 84, 117

Dorcatoma obtrita Logvinovskij ................................. 121

Dorcatoma punctulata Mulsant \& Rey ........ 82, 116, 118

Dorcatoma robusta Strand ........................ 116, 119, 120

Dorcatoma serra (Panzer) ........................................... 117

Dorcatoma setosella Mulsant \& Rey .................... 82, 83

Dorcatoma substriata Hummel ............................. 84, 117

Dorcatoma vaulogeri agenjoi Español .......................... 84

Dreposcia umbrina (Erichson) ....................................... 23

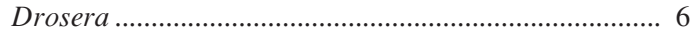

Drusilla canaliculata (Fabricius) ................................. 28

Drusilla canaliculata .................................................... 33

Dryopthorus corticalis (Paykull) ..................................... 33

Dyschirius globosus (Herbst) ...................................... 22

Dysstroma citrata conformalis (Prout) ....................... 213

Dysstroma citratum (Linnaeus) .......................... 213, 214

Dysstroma citratum kamtshadalarium ssp. n. Beljaev \&

Vasilenko ……………………………........ 213-216

Dysstroma infuscatum (Tengström) ......................... 216

Dysstroma pseudimmanatum (Heydemann) ....... 216, 232

Dysstroma truncata (Hufnagel) ................................. 232

Dytiscus dolabratus Paykull ..................................... 190

Dytiscus fuscus Linnaeus .......................................... 192

Dytiscus striatus Linnaeus ......................................... 190

Ecliptopera dimita (Prout) ........................................... 213

Ecliptopera silaceata leuca (Djakonov) ..... 212, 213, 217

Ecliptopera umbrosaria (Motschulsky) ..................... 213

Elachista (Aphelosetia) ..................................... 167, 168

Elachista (Dibrachia) .................................................. 168

Elachista (Elachista) .................................................. 168

Elachista adscitella Stainton ...................... 168, 170, 171

Elachista alpinella Stainton ............................... 164, 165 
Elachista bisulcella Duponchel........ $168,170,171,172$

Elachista chrysodesmella Zeller 168,171

Elachista cingillella (Herrich-Schäffer) . $167-186$ Elachista densicornella Hodgkinson ........... 169, 170, 173 Elachista eskoi Kyrki \& Karvonen... 163,164

Elachista excelsicola Braun 163-165

Elachista fasciola Parenti $169-171,174-183$

Elachista gangabella Zeller 168,171

Elachista heinemannii Frey 168-170

Elachista istanella Nielsen \& Traugott-Olsen

$168,170-172$

Elachista kilmunella Stainton

$163,165,166$

Elachista leifi Kaila \& Kerppola 163-166

Elachista megerlella auct., nec Hübner 168,171

Elachista metella Kaila. $170-172,174,176,181,182,183-186$

Elachista nedaella Traugott-Olsen $169-172,174,181,183,186$

Elachista obliquella Stainton

168,171

Elachista parasella Traugott-Olsen 163

Elachista pollutissima Staudinger

Elachista revinctella Zeller ...... ... 168 Elachista subalbidella Schläger ....................... 168, 170 Elachista sutteri Kaila ............... 170, 171, 174, 186-188 Elachista tinctella Sinev \& Sruoga ............................ 168 Elachista unifasciella Haworth ......................... 168, 171

Elachista ….................................................... 163, 167

Ematurga atomaria (Linnaeus) ........................... 201, 202

Ematurga atomaria krassnojarscensis Fuchs ............ 201

Ematurga atomaria minuta Heydemann .................... 201

Ematurga atomaria obsoletaria (Zetterstedt) ............ 201

Empetrum nigrum ........................................... 114, 131

Endocronartium pini .............................................. 44, 48

Enicmus transversus (Olivier) .................................... 32

Entephria caesiata (Denis \& Schiffermüller) ............ 210

Entephria Hübner ............................................... 13, 210

Entephria polata (Duponchel) ................................... 232

Entephria polata byssata (Aurivillius) ......................... 210

Entephria punctipes (Curtis) ............................ 210, 232

Eocatops lapponicus Szymczakowski .......................... 23

Eocatops lapponicus .................................................... 35

Epirrhoe alternata (Müller) .............................. 210, 232

Epirrhoe hastulata reducta (Djakonov) ............. 209, 232

Epirrhoe supergressa (Butler) ............................ 210, 232

Epirrhoe supergressa albigressa (Prout) ................... 210

Epirrhoe tristata (Linnaeus) ......................................... 232

Epirrita autumnata smetanini ssp. n. Beljaev .... 222, 223

Epuraea terminalis (Mannerheim) ................................ 31

Erica arborea ……............................................ 2, 2

Erica australis .................................................... 2, 2,

Eriophorum angustifolium ........................................ 166

Eriophorum russeolum ................................................ 131

Eriophorum vaginatum .............................................. 166

Eисопnиs claviger Mueller \& Kunze ............................. 23

Euconnus denticornis (Mueller \& Kunze) ..................... 24

Euconnus hirticollis (Illiger) .................................... 24

Euconnus maklinii (Mannerheim) ................................... 24

Euconnus pragensis (Machulka) .................................... 23
Euconnus wetterhallii (Gyllenhal) .............................. 24

Eulithis populata (Linnaeus) ........................................ 212

Eulithis prunata leucoptera (Djakonov) .................... 211

Eulithis testata (Linnaeus) ................................ 211, 212

Euphyia unangulata gracilaria (Bang-Haas) ............. 221

Euphyia unangulata renei Bryk ....................... 209, 221

Eupithecia abietaria debrunneata Staudinger............. 225

Eupithecia absinthiata (Clerck) ........................ 228, 233

Eupithecia actaeata praenubilata Inoue .................... 226

Eupithecia antaggregata Inoue ................................... 227

Eupithecia bohatschi Staudinger ............................... 225

Eupithecia castigata (Hübner) ...................................... 228

Eupithecia Curtis ................................................ 13, 17

Eupithecia gelidata hyperboreata Staudinger ............ 227

Eupithecia homogrammata Dietze ............................. 227

Eupithecia homogrammata kamtschatica Viidalepp \& Mironov ..................................................... 226, 232

Eupithecia indigata (Hübner) ..................................... 232

Eupithecia kurilensis mironovi ssp. n. Beljaev ...................

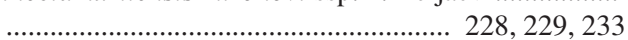

Eupithecia lariciata (Freyer) ..................................... 226

Eupithecia latipennis Hulst ............................................ 228

Eupithecia lvovskyi Mironov ...................................... 227

Eupithecia pimpinellata (Hübner) ....................... 226, 232

Eupithecia pseudosatyrata Djakonov ......................... 227

Eupithecia pusillata (Denis \& Schiffermüller) ........... 226

Eupithecia pygmaeata (Hübner) .......................... 17, 225

Eupithecia sobrinata (Hübner) ................................... 226

Eupithecia subfuscata (Haworth) ................................ 233

Eupithecia succenturiata exalbidata Staudinger ........ 229

Eupithecia ussuriensis Dietze ............................. 228, 233

Eupithecia veratraria geiserata Mironov .................. 229

Eupithecia veratraria Herrich-Schäffer ....................... 229

Eupithecia virgaureata Doubleday ................... 226, 233

Eupithecia zibellinata Christoph ................................. 225

Euplectus bonvouloiri rosae Raffray ............................ 29

Euplectus brunneus (Grimmer) .................................... 29

Euplectus decipiens Raffray ......................................... 29

Euplectus favveli Guillebeau ....................................... 29

Euplectus infirmus Raffray ............................................ 29

Euplectus karstenii (Reichhenbach) .............................. 29

Euplectus kirbii Danny ................................................. 29

Euplectus nanus (Reichhenbach) ................................. 29

Euplectus piceus Motschulsky ....................................... 29

Euplectus punctatus Mulsant ....................................... 29

Euplectus sanquineus Motschulsky ............................. 29

Euplectus signatus (Reichenbach) ............................... 29

Eupodes angardi Strandtmann \& Sømme ...................... 239

Eupodes tottanfjella Strandtmann .............. 236, 237, 239

Eupodes wisei Womersley \& Strandtmann ................. 239

Eupodes wisnesi Strandtmann \& Sømme ................... 239

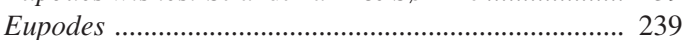

Euryporus picipes (Paykull) ......................................... 24

Euryusa castanoptera Kraatz ...................................... 29

Euryusa coarctata Märkel .......................................... 29

Euryusa optabilis Heer …………………………….... 29

Euryusa sinuata Erichson .............................................. 29

Eustroma reticulatum (Denis \& Schiffermüller) ........ 217 
Eustroma reticulatum chosenicola Bryk ...

Eustroma reticulatum obsoletum Djakonov

217

Eutheia linearis Mulsant

Eutheia scydmaenoides Stephens ................................. 23

Euthiconus conicicollis (Fair. \& Lab.) ......................... 23

Euura atra ........................................................... 135

Euura auritae Kopelke ..................................... 134-137

Euura cinereae Kopelke .................................... 134-137

Euura mucronata ..................................................... 136

Euura ...................................................... 135-137

Filipendula ulmaria ..................................................... 91

Fomes fomentarius ........................ 83, 84, 117, 119, 120

Fomitop sis pinicola …................................ 82, 117, 118

Formica ..................................................... 20, 35

Formica aquilonia Yarrow .................................. 34, 36

Formica cinerea Mayr ................................................ 34

Formica cunicularia ………………………………..... 34

Formica exsecta Nylander ........................................... 34

Formica fusca (Linnaeus) ............................................... 34

Formica gagatoides Ruzsky .......................................... 34

Formica lemani Bondroit ............................................. 34

Formica lugubris Zetterstedt .................................. 34, 36

Formica nigricans Emery ............................................ 34

Formica polyctena Förster .................................... 34, 36

Formica pratensis Retzius ..................................... 34, 36

Formica rufa Linnaeus .................................... 33, 34, 36

Formica rufibarbis Fabricius ....................................... 34

Formica sanguinea Latreille ........................................ 34

Formica suecica Adlerz ............................................ 34

Formica truncorum Fabricius ......................................... 34

Formica uralensis Ruzsky ............................................ 34

Gabrius nigritulus (Gravenhorst) .................................. 24

Gabrius osseticus (Kolenati) ....................................... 24

Gabrius sphagnicola (Sjöberg) .................................... 24

Gabrius splendidulus (Gravenhorst) ............................ 24

Gagitodes sagittatus albiflua (Prout) ......................... 224

Ganoderma applanatum ........................... 117, 119, 120

Ganoderma ……..................................................... 83

Geometra papilionaria herbacearia Ménétriès.......... 202

Geostiba circellaris (Gravenhorst) ................................ 27

Geranium sylvaticum .................................................... 91

Glaenocorisa cavifrons (Thomson) ........................ 86, 87

Glaenocorisa propinqua (Fieber) .................................. 87

Glaenocorisa quadrata Walley .................................... 87

Glaenocorisa ........................................................... 87

Globicornis emarginata (Gyllenhal) ............................ 31

Gnaphalium norvegicum .......................................... 113

Gnaphalium sylvaticum ......................................... 113

Gnathonchus rotundatus (Kugelann) .......................... 30

Gongylidium …………………………………..... 131

Greniera brachiata ................................................... 157

Greniera ................................................. 155, 157

Gyrohypnus angustatus (Stephens) ............................ 24

Gyrohypnus atratus (Heer) ......................................... 24

Gyrohypnus scoticus (Joy) .......................................... 24

Halia halituaria (Guenée) .......................................... 200

Hapalaraea nigra (Gravenhorst)

... 25

Haplodrassus signifer (C. L. Koch) .......................... 131

Haploglossa gentilis (Märkel) ....................................... 27

Haploglossa marginalis (Gravenhorst) ....................... 27

Haploglossa picipennis (Gyllenhal) .............................. 27

Haploglossa villosula (Stephens) ................................... 27

Harkeria Cameron ....................................................... 161

Hesperocorixa atopondota (Hungerford) ...................... 86

Hesperocorixa harrisii (Uhler) ..................................... 86

Hesperocorixa interrupta (Say) ................................... 86

Hesperocorixa kennicottii (Uhler) .................................. 86

Hesperocorixa lobata (Hungerford) .............................. 86

Hesperocorixa lucida (Abbott) ....................................... 86

Hesperocorixa martini (Walley) .................................. 86

Hesperocorixa michiganensis (Hungerford) ................. 86

Hesperocorixa minor (Abbott) ....................................... 86

Hesperocorixa minorella (Hungerford) ........................ 86

Hesperocorixa nitida (Fieber) ....................................... 86

Hesperocorixa obliqua (Hungerford) ............................. 86

Hesperocorixa scabricula (Walley) ............................... 86

Hesperocorixa semilucida (Walley) .............................. 86

Hesperocorixa vulgaris (Hungerford) .......................... 86

Hetaerius ferrugineus (Olivier) ..................................... 31

Heterobasidion ………………………………...... 44, 48

Heterothera taigana sounkeana (Matsumura) ... 217, 232

Heterothops dissimilis (Gravenhorst) ........................... 24

Heterothops niger Kraaz ............................................ 24

Heterothops praevius Erichson .................................... 24

Hilaira nubigena Hull ................................................ 131

Hilara albitarsis ........................................................... 75

Hilara angusticauda Oldenberg ................................. 65

Hilara bohemica Straka ............................................... 75

Hilara borealis Oldenberg .................................... 66-71

Hilara brevivittata Macquart ........................................... 68

Hilara caerulescens Oldenberg ....................... 66, 71-73

Hilara calinota Collin .......................................... 68, 65

Hilara chorica (Fallén) .......................................... 66, 67

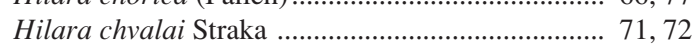

Hilara coracina Oldenberg .............................. 66, 73-75

Hilara czernyi Strobl ..................................... 66, 67, 69

Hilara dalmatina Strobl .............................................. 73

Hilara dimidiata Strobl ............................................... 73

Hilara femorata Loew .................................................. 77

Hilara interstincta (Fallén) ............................................ 73

Hilara lacteipennis Strobl ............................................ 68

Hilara lapponica Chvála ....................................... 67-71

Hilara lasiochira Strobl ............................................... 77

Hilara longivittata Zetterstedt .......................... 68, 69, 74

Hilara medeteriformis Collin ............................ 65, 68, 75

Hilara monedula Collin ............................................... 74

Hilara morata Collin ................................................... 65

Hilara nana Macquart ................................................. 66

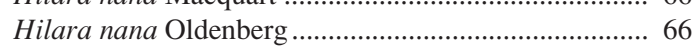

Hilara nigrohirta Collin ............................................ 72

Hilara perversa Oldenberg ............................... 66, 75-77

Hilara platyura Loew ................................................... 77

Hilara pruinosa Wiedemann ................................... 68, 68

Hilara pseudochorica Strobl ......................................... 68

Hilara quadrifaria Strobl ..................................... 74,75 
Hilara woodi Collin

68,69

Hilara

$65,66,74,75$

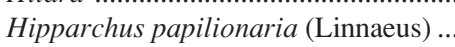
202

Hister helluo Truqui .................................................. 30

Hister unicolor Linnaeus .............................................. 30

Hydrelia flammeolaria (Hufnagel) .............................. 231

Hydriomena furcata (Thunberg) ....................... 218, 232

Hydriomena impluviata djakonovi ssp. n. Beljaev

218,219

Hydriomena ruberata (Freyer) ................................... 232

Hypnogyra glabra (Nordmann) ...................................... 24

Hypocoprus hispanis ................................................. 140

Hypocoprus lathridioides Motschulsky ....................... 32

Hypocoprus latridioides (Motschulsky) ..... 139, 140, 142

Hypocoprus quadricollis Reitter ................ 139, 140, 142

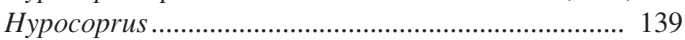

Ilyobates nigricollis (Paykull) ........................................ 27

Ilyobates subopacus Palm ............................................ 27

Inonotus cuticularis ………………………………..... 83

Inonotus radiatus ................................................ 117, 119

Inonotus rheades ….......................................... 117, 119

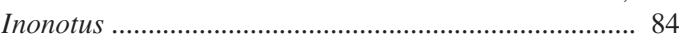

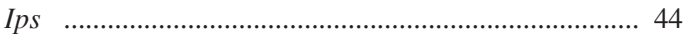

Ischnoglossa prolixa (Gravenhorst) ............................. 26

Ischnosoma bergrothi (Hellen) ........................................ 25

Isturgia Hübner .......................................................... 200

Itame brunneata (Thunberg) ...................................... 199

Itame fulvaria (Villers) ................................................ 199

Itame loricaria (Eversmann) ........................................ 199

Itame wauaria (Linnaeus) ........................................... 199

Juncus gerardi ......................................................... 114

Juniperus oxycedrus ………………………………..... 2

Juniperus thurifera …………………………………..... 2

Kikimora Eskov ......................................... 129, 131

Kikimora palustris Eskov ...................................... 129-132

Laetiporus sulphureus ........................................ 82, 117

Lamprinodes saginatus (Gravenhorst) ……………..... 25

Lampropteryx otregiata Metcalfe ....................... 211, 232

Lampropteryx suffumata (Denis \& Schiffermüller) ... 211

Lampropteryx suffumata defumata (Stichel) .............. 211

Lamptopteryx minna (Butler) ...................................... 232

Larentia ferrugata (Clerck) ........................................ 207

Larentia hastata (Linnaeus) ....................................... 220

Larentia immanata (Haworth) ...................................... 213

Larentia lugubrata albidior (Alpheraky) ................... 220

Larentia sociata (Borkhausen) .................................... 210

Larentia suffumata minna (Butler) .............................. 211

Larentia tristata (Linnaeus) ....................................... 209

Larix .................. 131, 199, 201, 217, 222, 225, 226, 227

Lasius ................................................................... 35

Lasius alienus (Förster) ................................................. 34

Lasius brunneus (Latreille) .............................................. 34

Lasius flavus (Fabricius) ................................................. 34

Lasius fuliginosus (Latreille) ................................... 33, 34

Lasius mixtus (Nylander) .............................................. 34

Lasius niger (Linnaeus) ................................................. 34

Lasius umbratus (Nylander) .......................................... 34

Leiestes seminigra (Gyllenhal) .................................... 32
Leiopus punctulatus (Paykull) ....................................... 48

Leptacinus formicetorum Märkel ................................. 24

Leptorhaptus aukt ..................................................... 57

Leptothorax ………………………………………..... 35

Leptothorax acervorum (Fabricius) .............................. 34

Leptusa ruficollis (Erichson) ....................................... 29

Leucobrephos middendorffi middendorffi (Ménétriès, 1858)

198

Leucobrephos middendorffi ussuriensis (Moltrecht, 1909)

198

Leucocytozoon lovati

Liocola marmorata (Fabricius) ..................................... 31

Liogluta alpestris (Heer) ............................................. 27

Liogluta longiuscula (Gravenhorst) ............................. 27

Liogluta micans (Mulsant \& Rey) ................................ 27

Loensia variegata (Latreille) ................................. 58, 59

Lomaspilis marginata (Linnaeus) ................................ 198

Lomaspilis marginata amurensis (Hedemann, 1881) ........

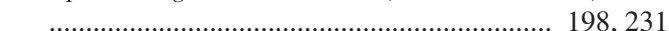

Lomaspilis marginata opis (Butler) .............................. 198

Lomaspilis opis (Butler) ................................... 198, 231

Lomechusa emarginata (Paykull)................................ 29

Lomechusa paradoxa Gravenhorst ................................ 29

Lomechusa pubicollis Brisout de B...……………….... 29

Lomechusoides inflatus (Zetterstedt) .............................. 29

Lomechusoides strumosus (Fabricius) .......................... 28

Lomechusoides wellenii (Palm) .................................... 29

Lucilia illustris .................................................... 100-102

Lucilia magnicornis (Siebke) .................................... 101

Lucilla silvarum Meig. .................................................... 99

Lycaena phlaeas (L.) .................................................... 17

Lycia hirtaria (Clerck) ............................................. 200

Lycia pomonaria (Hübner) ............................................ 201

Lyctus linearis (Goeze) ................................................ 31

Lygris populata (Linnaeus) ......................................... 212

Lygris prunata leucoptera (Djakonov) ........................ 211

Lygris testata (Linnaeus) ............................................ 211

Lyprocorrhe anceps (Erichson) ................................... 28

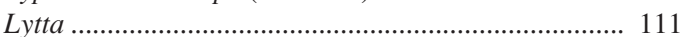

Macaria brunneata brunneata (Thunberg) ................. 199

Macaria halituaria (Guenée) ...................... 199, 200, 231

Macaria loricaria (Eversmann) .................................. 199

Macaria signaria (Hübner) ........................................ 199

Macaria wauaria (Linnaeus) ....................................... 231

Macrohynnis ferrugineus Macek ..................... 51, 53-55

Macrohynnis Förster ........................................ 51, 52, 57

Macrohynnis fragilis (Nixon) ................................ 51-57

Macrohynnis lepidus Mayr ...................................... 51-57

Macrohynnis rufiventer Kieffer ............................ 51, 52

Maianthemum bifolium ................................................. 91

Margarinotus merdarius (Hoffmann) ........................... 30

Martania taeniatum obsoletum (Djakonov) .................. 224

Mecynargus sphagnicola (Holm) .............................. 131

Medon apicalis (Kraatz) .............................................. 25

Medon castaneus (Gravenhorst) .................................... 25

Medon fusculus (Mannerheim) ....................................... 25

Medon rufiventris (Nordmann) ...................................... 25

Megaselia brevicostalis (Wood) ......................... 124-127 
Megaselia diversa (Wood)

125,126

Megaselia giraudii (Egger)

$125-127$

Megaselia minor ................................................... 126

Megaselia nigriceps ............................................... 126

Megaselia unicolor ................................................... 126

Megaselia verralli (Wood) ................................ 124-126

Megaselia woodi (Lundbeck) ............................. 125, 126

Megaselia zonata ................................................... 126

Megaselia ............................................... 124, 126, 127

Megasternum obscurum (Marsham) ............................ 22

Meliceria tragardhi Palm ............................................ 29

Meloe …..................................................................... 111

Meotica exilis (Knoch) ................................................. 27

Metacnephia lyra (Lundström) ........................... 155-157

Meteorus corax Marshall ............................................ 46

Metopina oligoneura ................................................. 126

Metopina ………………………………….... 127

Micrambe abietis (Paykull) ........................................... 32

Micridium halidaii (Matthews) .................................... 22

Microscydmus minimus (Chaudoir) ............................... 23

Microscydmus nanus (Schaum) .................................... 23

Millium effusum ............................................................ 175

Miota Förster ………………………………………. 57

Monochamus galloprovincialis (Olivier) ............... 44, 46

Monoctonus allisoni Pike \& Stary .............................. 161

Monoctonus caricis .................................................... 161

Monoctonus leclanti sp. $\mathrm{n}$. Tomanovic \& Stary . 160, 161

Monoctonus nervosus (Haliday) ......................... 160, 161

Monoctonus pacificus Pike \& Stary ........................... 161

Monoctonus paulensis Asmead .................................. 161

Monoctonus ......................................................... 159

Monotoma angusticollis (Gyllenhal) ........................... 32

Monotoma caucasicum Kolennati .............................. 142

Monotoma conicicollis Aube ....................................... 31

Monotoma longicollis (Gyllenhal) ................................ 32

Monotoma picipes Herbst ............................................ 32

Monotoma quadrifoveolata Aubé ................................ 31

Mycetaea subterranea (Fabricius) ................................ 32

Mycetophagus guadriguttatus Mueller .......................... 33

Mycetoporus lepidus (Gravenhorst) .............................. 25

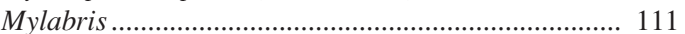

Myrmechixenus subterraneus Chevrolat ...................... 33

Myrmecinomus hochuthii Chaudior .......................... 142

Myrmetes paykulli Kanaar .............................................. 30

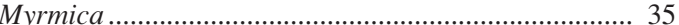

Myrmica rubra (Linnaeus) ........................................... 34

Myrmica ruginodis Nylander ...................................... 34

Myrmica rugulosa Nylander......................................... 34

Myrmica sabuleti Meinert ............................................ 34

Myrmica scabrinodis Nylander ..................................... 34

Myrmica sulcinodis Nylander ...................................... 34

Nanorchestes bifurcatus Strandtmann ........ 236, 237, 239

Nanorchestes brekkeristae Strandtmann \& Sømme ... 236

Nanorchestes ...................................................... 237

Nemadus colonoides (Kraaz) ........................................ 23

Nevraphes angulatus (Müller \& Kunze) ....................... 23

Nevraphes elongatulus (Müller \& Kunze) .................... 23

Nevraphes plicicollis Reitter

23
Nevraphes talparum Lokay .......................................... 23

Nicrophorus vespilloides Hebst. ................................. 102

Noctua cordigera Thunberg .............................................. 1

Noctua myrtilli L. ........................................................ 1

Nothopteryx polycommata (Denis \& Schiffermüller) 231

Nudobius lentus (Gravenhorst) ..................................... 24

Ocalaea badia Erichson ................................................. 27

Oligota muensteri Bernhauer ...................................... 29

Oligota pusillima (Gravenhorst) .................................... 29

Oligota tantilla Mennerheim .......................................... 29

Oligota uralensicola Kangas ......................................... 29

Omalium caesum Gravenhorst ........................................ 25

Opatrum sabulosum (Linnaeus) ..................................... 33

Operophtera ……………………………………..... 13

Oporinia autumnata (Borhausen) ............................... 222

Orthoperus punctulatus Reitter .................................... 32

Othius angustus Stephens ........................................... 25

Othius myrmecophilus Kiesenwetter ............................. 25

Othius punctulatus (Goeze) ........................................... 25

Ousipalia caesula (Erichson) ......................................... 27

Oxylaemus variolosus (Dufour) ................................... 32

Oxypoda abdominalis (Mannerheim) ............................ 26

Oxypoda acuminata (Stephens) .................................... 26

Oxypoda advena Mäklin ............................................. 26

Oxypoda annularis (Mannerheim) .................................. 26

Oxypoda arborea Zerche ................................................ 26

Oxypoda brachyptera (Stephens) ................................. 26

Oxypoda exoleta Erichson ........................................... 26

Oxypoda ferruginea Erichson ........................................ 26

Oxypoda flavicornis Kraatz ........................................ 26

Oxypoda formiceticola Märkel ......................................... 26

Oxypoda haemorrhoa Mannerheim ............................ 26

Oxypoda hansseni Strand ............................................. 26

Oxypoda longipes Mulsant \& Rey ................................ 26

Oxypoda opaca (Gravenhorst) ..................................... 26

Oxypoda pratensicola Lohse ......................................... 26

Oxypoda recondita Kraatz ............................................. 26

Oxypoda rugicollis Kraatz ............................................ 26

Oxypoda serpentata Kangas .......................................... 26

Oxypoda soror Thomson .............................................. 26

Oxypoda spectabilis Märkel ........................................... 26

Oxypoda testacea Erichson .......................................... 26

Oxypoda togata Erichson ............................................ 26

Oxypoda umbrata (Gyllenhal) ........................................ 26

Oxypoda vittata Märkel ................................................ 26

Pachyneuron aphidis (Bouché) .................................. 162

Palmacorixa buenoi Abbott …............................. 86

Palmacorixa gillettei Abbott ........................................ 86

Palmacorixa nana nana Walley ................................... 86

Palorus depressus (Fabricius) ...................................... 33

Paraplatyptilia pterodactyla (Walker) ....................... 128

Pardosa atrata (Thorell) ........................................... 131

Pardosa hyberborea (Thorell) ..................................... 131

Parocyusa rubicunda (Erichson) .................................. 26

Paromalus flavicornis (Herbst) .................................... 30

Pelecopsis mengei (Simon) ........................................ 131

Peltis grossa .......................................................... 82

Pentaphyllus testaceus (Hellwig) .................................. 33 
Perizoma custodiata (Guenée)

Perizoma haasi (Hedemann)

232

224

Perizoma sagittatum (Fabricius) ................................ 224

Phalacrotophora .................................................... 127

Phasiane clathrata (Linnaeus) ................................... 198

Phellinus cinereus ............................................ 117, 119

Phellinus igniarius ............................................ 117, 119

Phellinus nigricans ........................................... 117, 119

Phellinus populicola …………………................ 117, 119

Phellinus tremulae ........................................... 117, 119

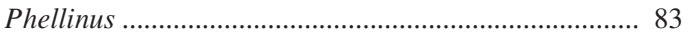

Philonthus ventralis (Gravenhorst) .............................. 24

Phora penicillata …................................................. 126

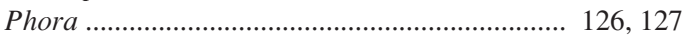

Phyllocolpa ……........................................... 135

Picea abies .............................................. 59, 82, 90, 91

Picea …................................. 48, 207

Pilosodorcatoma Borowski ........................................... 82

Pinus abies ............................................................. 90

Pinus halepensis ............................................................... 2

Pinus nigra ............................................................... 2

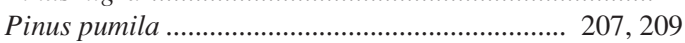

Pinus sylvestris .................................................. 2, 2, 123

Pinus ................................................... 48

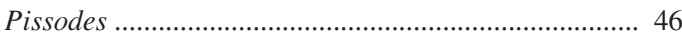

Pityogenes chalcographus (L.) .................................... 43

Pityogenes quadridens (Hartig) ..................................... 44

Platycis cosnardi (Chevrolat) ....................................... 31

Platycis minuta (Fabricius) ............................................. 31

Platydracus fulvipes (Scopoli) ...................................... 24

Platydracus latebricola (Gravenhorst) .......................... 24

Platydracus stercorarius (Olivier) ............................... 24

Platynaspis luteorubra (Goeze) ..................................... 32

Platystethus arenarius (Fourcroy) ................................. 25

Plectophloeus nitidus (Fairmaire) ............................... 29

Plegaderus caesus (Herbst) ........................................ 30

Plegaderus dissectus Erichson .................................... 30

Poecilopsis pomonaria (Hübner) ................................. 201

Poeciloptilia cingillella Herrich-Schäffer ................... 173

Polyergus rufescens (Latreille) ..................................... 34

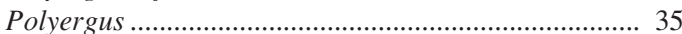

Polygonum viviparum .................................................. 90

Polygraphus …………………………………..... 43

Polythrena coloraria pallida Djakonov ...................... 230

Pontania ....................................................... 135, 136

Populus tremula …………………………….... 59, 94

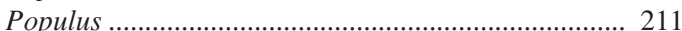

Poromniusa procidua (Erichson) ................................ 27

Porotachys bisulcatus (Nicolai) ................................... 22

Potosia cuprea (Fabricius) .......................................... 31

Praestigia groenlandica Holm ................................ 131

Protereunetes maudae Strandtmann ............................. 236

Protocalliphora chrysorrhoea Meig. .......................... 99

Protocalliphora nuortevai Grun. ............................... 101

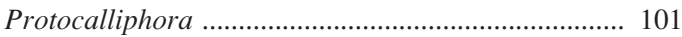

Protophormia atriceps Zett. ........................................ 101

Protophormia terraenovae R.-D. ...................... 100-102

Pseudophilotes baton (Bergsträsser)
Psocidus flavonimbatus (Rostock)

.. 59

Psocidus ......... 58, 61

Psocus flavonimbatus Rostock ............................... 58-62

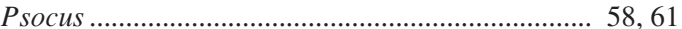

Psychophora sabini (Kirby) ............................... 223, 224

Ptenidium formicetorum Kraatz .................................. 22

Ptenidium gressneri Erichson ....................................... 22

Ptenidium laevigatum Erichson ..................................... 22

Ptenidium pusillum (Gyllenhal) ................................... 22

Ptenidium turgidum Thomson ....................................... 22

Pteryx suturalis (Heer) ................................................ 22

Ptilium modestum Wankowicz ..................................... 22

Ptilium myrmecophilum (Allibert) ............................... 22

Ptinus subpilosus Sturm .............................................. 31

Ptomaphagus sericatus (Chaudoir) .............................. 23

Ptycta chubsugulensis Günther ..................................... 61

Pygmaena fusca (Thunberg) .................................... 200

Quedius boops (Gravenhorst) .......................................... 24

Quedius brevicornis Thomson ..................................... 24

Quedius brevis Erichson ........................................ 24, 33

Quedius fuliginosus (Gravenhorst) ............................... 24

Quedius invreai Gridelli ................................................ 24

Quedius maurus (Sahlberg) .......................................... 24

Quedius mesomelinus (Marsham) ................................... 24

Quedius microps (Gravenhorst) ..................................... 24

Quedius ochripennis (Ménétriés) ................................. 24

Quedius scitus (Gravenhorst) ....................................... 24

Quedius truncicola Fair. \& Lab..................................... 24

Quercus faginea ............................................................. 2

Quercus pyraenica .................................................. 2, 6

Quercus rotundifolia ...................................................... 2

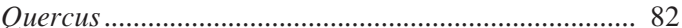

Ramphocorixa acuminata (Uhler) .................................. 86

Rhagium inquisitor (L.) ......................................... 44, 46

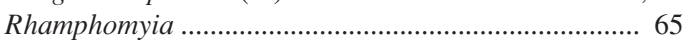

Rheumaptera hastata (Linnaeus) ....................... 220, 221

Rheumaptera subhastata commixta (Matsumura) .... 220, 221

Rhodostrophia .......................................................... 13

Robertus lyrifer Holm .................................................. 131

Rubus chamaemorus ……………………………….... 131

Sagina nodosa .................................................. 114, 115

Salix alba ……................................................... 135

Salix aurita ...................................................... 134-137

Salix caprea ...................................................... 135-137

Salix cinerea …............................................. 134-137

Salix fragilis ........................................................... 135

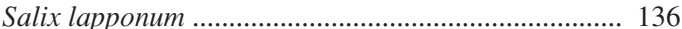

Salix myrsinifolia .......................................................... 136

Salix repens .................. 114

Salix sachalinensis .................................... 211, 212, 218

Salix strakeana ….......................................... 135, 137

Salix …........................ 82, 94, 131, 211, 213, 219, 223

Saulcyella schmidtii (Märkel) ........................................ 29

Sciastes Bishop \& Crosby ............................................ 131

Sciastes truncatus (Emerton) ........................................ 131

Scopaeus laevigatus (Gyllenhal) .................................... 25

Scopaeus minutus Erichson ............................................ 25

Scopaeus pusillus Kiesenwetter .................................. 25 
Scopula frigidaria frigidaria (Möschler) .................... 203

Scorpioteleia Ashmead ............................................... 57

Scraptia fuscula Mueller ............................................. 33

Scydmaenus hellwigii (Herbst) ...................................... 24

Scydmaenus perrisii (Reitter) ....................................... 24

Scydmaenus rufus Müller \& Kunze ............................... 24

Scydmaenus tarsatus Muller \& Kunze ......................... 24

Scydmographes helvolus (Schaum) .............................. 23

Scydmoraphes minutus (Chaudoir) ................................. 23

Scythris albisaxella Nupponen \& Nupponen ..... 148, 149

Scythris alceella sp. n. Junnilainen ..................... 146, 147

Scythris arkaimensis Bengtsson ........................ 148, 149

Scythris derrai Bengtsson ......................................... 150

Scythris dissimilella (Herrich-Schäffer) ...................... 150

Scythris kullbergi Bengtsson .................................... 148

Scythris lagomorphella sp. n. Junnilainen ........... 148, 149

Scythris tabelli sp. n. Junnilainen ...................... 149-152

Scythris tenuivittella (Stainton) .................................. 150

Scythris terekholensis Bengtsson ............................... 148

Scythris traugotti Bengtsson ........................................ 150

Scythris vartianae Kasy ............................................ 150

Scythris vittella Costa ............................................... 150

Selenia bilunaria (Esper) .......................................... 200

Selenia bilunaria alpestris Wehrli............................. 200

Selenia bilunaria minima Strand ................................ 200

Selenia dentaria (Fabricius) ....................................... 200

Selidosema brunnearium (Villers) .......................... 14-16

Selidosema pulmarium (Denis \& Schiffermüller) .. 14-16

Selidosema .............................................................. 17

Semiothisa (Chiasmia) clathrata (Linnaeus).............. 198

Semiothisa signaria (Hübner) ....................................... 199

Semljicola faustus (O. P.-Cambridge) ......................... 131

Sepedophilus immaculatus (Stephens) ........................... 25

Sepedophilus marshami (Stephens) ............................... 25

Sepedophilus testaceus (Fabricius) ............................... 25

Sigara alternata (Say) .................................................. 86

Sigara bicoloripennis (Walley) .................................... 86

Sigara compressoidea (Hungerford) ………………..... 86

Sigara conocephala (Hungerford) ................................ 86

Sigara decorata (Abbott) ............................................. 86

Sigara decoratella (Hungerford) ................................... 86

Sigara defecta Hungerford \& Sailer ............................... 86

Sigara dolabra Hungerford \& Sailer ............................. 86

Sigara douglasensis (Hungerford) ................................ 86

Sigara fallenoidea (Hungerford) ................................. 86

Sigara gordita (Abbott) ......................................... 86, 87

Sigara grossolineata Hungerford ................................. 86

Sigara hubbelli (Hungerford) ....................................... 86

Sigara johnstoni Hungerford ........................................ 86

Sigara knighti Hungerford ............................................ 86

Sigara lineata (Forster) ............................................... 86

Sigara mackinacensis (Hungerford) ............................... 86

Sigara macropala (Hungerford) ……………………... 86

Sigara mathesoni Hungerford ....................................... 86

Sigara modesta (Abbott) .............................................. 86

Sigara mullettensis (Hungerford) .................................. 87

Sigara ornata (Abbott) ................................................... 87

Sigara penniensis (Hungerford) .................................... 87
Sigara quebeckensis (Walley) ...................................... 87

Sigara signata (Fieber) .................................................. 87

Sigara solensis (Hungerford) ....................................... 87

Sigara stigmatica (Fieber) ............................................. 87

Sigara transfigurata (Walley) ...................................... 87

Sigara trilineata (Provancher) .................................... 87

Sigara variabilis (Hungerford) ....................................... 87

Sigara virginiensis Hungerford ................................... 87

Sigara zimmermanni (Fieber) ....................................... 87

Silene maritima 114

Simulium (Eusimulium) aureum Fries ................ 155-157

Simulium (Nevermannia) bicorne Dorogostaisky, Rubtsov

\& Vlasenko ............................................ 155, 157

Simulium (Nevermannia) vernum Macquart ...... 155-157

Simulium (Parabyssodon) transiens Rubtsov ... 155-157

Simulium (Simulium) truncatum (Lundström) ............ 155

Simulium cryophilum (Rubtsov) ................................. 155

Spargania luctuata albidior (Alpheraky) ................... 220

Spavius glaber (Gyllenhal) ........................................... 32

Sphagnum fuscum ..................................................... 130

Sphagnum lindbergii ................................................ 131

Sphagnum russowii .................................................... 131

Sphagnum ………….................................... 6, 130, 131

Spiniphora maculata ................................................. 127

Staphylinus erythropterus Linnaeus .............................. 24

Stenamma ……………………………………….. 35

Stenamma westwoodii Westwood ................................. 34

Stenichnus bicolor (Denny) ............................................ 23

Stenichnus collaris (Mueller \& Kunze) .......................... 23

Stenichnus godarti (Latreille) ................................... 23

Stenichnus pusillus (Müller \& Kunze) ........................... 23

Stenichnus scutellaris (Müller \& Kunze) ...................... 23

Stenoptilodes petradactyla (Wilkinson) ....................... 128

Stenus aterrimus Erichson .......................................... 25

Stenus crassus Stephens ............................................... 25

Stephotethus rugicollis (Olivier) .................................. 32

Stichoglossa semirufa (Erichson) .................................... 26

Sunius bicolor (Olivier) ............................................ 25

Sunius melanocephalus (Fabricius) .............................. 25

Symbiotes gibberosus (Lucas) ...................................... 32

Symbiotes latus Redtenbacher ..................................... 32

Syntomus truncatellus (Linnaeus) ................................. 22

Tachinus fimetarius Gravenhorst ................................. 25

Tachinus marginellus (Fabricius) .................................. 25

Tachinus rufipes (Linnaeus) ....................................... 25

Tachyporus chrysomelinus (Linnaeus) .......................... 25

Tachyporus corpulentus Sahlberg ............................... 25

Tachyporus hypnorum (Fabricius) ............................... 25

Tachyporus nitidulus (Fabricius) .................................. 25

Tachyporus obtusus (Linnaeus) .................................... 25

Tachyporus scitulus (Erichson) .................................... 25

Tachyusida gracilis (Erichson) ...................................... 29

Tapinoma erraticum Latreille ........................................ 34

Tapinoma ……………………………………..... 35

Teredus cylindricus (Olivier) ...................................... 32

Tetramorium .................................................................. 35

Tetramorium caespitum (Linnaeus) .............................. 34

Tetrao tetrix L....................................................... 154 
Tetropium gabrieli Weise

Tetropium

Thamiaraea hospita (Märkel).

Thera variata (Denis \& Schiffermüller).

Thera variata bellisi Viidalepp

Thiasophila angulata (Erichson)

Thiasophila bercionis Bernhauer

Thiasophila canaliculata Mulsant \& Rey .................... 26

Thiasophila inquilina Märkel ........................................ 27

Thiasophila lohsei Zerche .............................................. 27

Thiasophila wockii (Schneider) ..................................... 26

Timandra puziloi Erschoff ........................................... 202

Timandra rectistrigaria (Eversmann) ........................ 202

Tomicus minor (Hartig) .................................................. 44

Tomicus piniperda $(\mathrm{L}$.$) ....................................................... 44$

Trichadenotecnum germanicum Roesler ................ 61-62

Trichius fasciatus (Linnaeus) ........................................ 31

Trichocorixa borealis Sailer .......................................... 87

Trichocorixa calva (Say) ........................................... 87

Trichocorixa louisianae Jaczewski .............................. 87

Trichocorixa macroceps (Kirkaldy) .............................. 87

Trichocorixa sexcincta (Campion) ................................. 87

Trichocorixa verticalis (Fieber) ..................................... 87

Trichocorixa verticalis fenestrata (Walley) ........... 87, 88

Trichocorixa verticalis sellaris (Abbott) ....................... 87

Trichocorixa verticalis verticalis (Fieber) .............. 87, 88

Trichonyx sulcicollis (Reichenbach) ............................ 30

Trichophya pilicornis Gyllenhal) ................................. 25

Trichopteryx polycommata grisea (Djakonov) .......... 231

Trifolium pratense .................................................. 91-95

Trifolium repens .......................................................... 91

Trimium brevicorne (Reichenbach) ............................. 30

Triphleba autumnalis .................................................. 127

Triphleba trinervis ……...................................... 127

Triphleba ............................................... 126, 127

Trollius europaeus ....................................................... 91

Trypocalliphora braueri Hend. ................................. 101

Trypodendron ………………………………...... 42, 43

Tychus niger (Paykull) ............................................... 30

Tydeus erebus Strandtmann ............................... 236, 237

Tydeus setsukoe Strandtmann .............................. 236, 237
Tyrus mucronatus (Panzer) ........................................... 30

Upocorpus latridioides Motschulsky ......................... 142

Vaccinium microcarpum ........................................... 131

Vaccinium uliginosum ................................................. 131

Vaccinium vitis-idea .................................................. 131

Venusia cambrica Curtis .................................. 230, 231

Walckenaeria nudipalpus (Westring) ........................ 131

Xantholinus laevigatus Jacobson ................................... 25

Xantholinus linearis (Olivier) ....................................... 25

Xantholinus meyeri Drugmand ........................................ 25

Xantholinus tricolor (Fabricius) ..................................... 25

Xanthorhoe asiatica (Staudinger) ............................... 206

Xanthorhoe biriviata angularia (Leech) .................... 203

Xanthorhoe decoloraria (Esper)........................ 204, 209

Xanthorhoe derzhavini derzhavini (Djakonov) .......... 208

Xanthorhoe designata(Hufnagel) ................................ 204

Xanthorhoe ferrugata malaisei (Djakonov) ................ 207

Xanthorhoe fluctuata (Linnaeus) .................................. 207

Xanthorhoe fluctuata malleola Inoue ........................ 208

Xanthorhoe fluctuata simushira (Bryk) ...................... 208

Xanthorhoe insperata (Djakonov) .............................. 206

Xanthorhoe kamtshatica (Djakonov) .................. 204, 208

Xanthorhoe montanata (Denis \& Schiffermüller) ...... 232

Xanthorhoe okhotinaria sikhotenaria ssp. n. Beljaev \&

Vasilenko ............................................................ 207

Xanthorhoe okhotinaria sp. n. Beljaev \& Vasilenko .........

204, 206, 232

Xanthorhoe rectantemediana (Wehrli)...................... 203

Xanthorhoe spadicearia (Denis \& Schiffermüller) ............

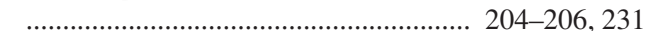

Xenotoma Förster ........................................................ 57

Xorides irrigator (Fabricius) ....................................... 46

Xylodromus affinis (Gerhardt) ........................................ 25

Xylodromus depressus (Gravenhorst) ........................... 25

Zyras cognatus (Märkel) .............................................. 28

Zyras collaris (Paykull) ................................................. 28

Zyras funestus (Gravenhorst) ....................................... 28

Zyras humeralis (Gravenhorst) .................................... 28

Zyras laticollis (Märkel) .............................................. 28

Zyras limbatus (Paykull) ............................................... 28

Zyras lugens (Gravenhorst) …............................... 28 